\title{
Existence and uniqueness of positive large solutions to some cooperative elliptic systems
}

\author{
Jorge García-Melián* \\ Departamento de Análisis Matemático \\ Universidad de La Laguna, 38271 La Laguna, Tenerife, SPAIN \\ e-mail: jjgarmel@ull.es \\ Antonio Suárez ${ }^{\dagger}$ \\ Departamento de Ecuaciones Diferenciales y Análisis Numérico \\ Universidad de Sevilla, 41012 Sevilla, SPAIN \\ e-mail: suarez@numer.us.es
}

\begin{abstract}
In this work we consider positive solutions to cooperative elliptic systems of the form $-\Delta u=\lambda u-u^{2}+b u v,-\Delta v=\mu v-v^{2}+c u v$ in a bounded smooth domain $\Omega \subset \mathbb{R}^{N}(\lambda, \mu \in \mathbb{R}, b, c>0)$ which blow up on the boundary $\partial \Omega$, that is $u(x), v(x) \rightarrow+\infty$ as $\operatorname{dist}(x, \partial \Omega) \rightarrow 0$. We show existence and nonexistence of solutions, and give sufficient conditions for uniqueness. We also provide an exact estimate of the behaviour of the solutions near the boundary in terms of $\operatorname{dist}(x, \partial \Omega)$.
\end{abstract}

2000 Mathematics Subject Classification. 35J55, 35B40.

Key words. Cooperative systems - Boundary blow up - Sub and supersolutions - Distance function

*Supported by MCYT under contracts \# BFM2000-0797 and \# BFM2001-3894.

${ }^{\dagger}$ Supported by MCYT under contract \# BFM2000-0797. 


\section{Introduction}

Boundary blow up elliptic problems have been widely treated in the literature. They generally take the form

$$
\left\{\begin{array}{ccc}
-\Delta u=f(x, u) & \text { in } & \Omega \\
u=+\infty & \text { on } & \partial \Omega
\end{array}\right.
$$

where the boundary condition is to be understood as $u(x) \rightarrow+\infty$ as $d(x):=$ $\operatorname{dist}(x, \partial \Omega) \rightarrow 0$. Their history begins with works of Bieberbach [2], Keller [15] and Osserman [21]. Recently they have received a great deal of attention, for instance in [1], [5], [6], [11] or [20] (see the list of references in [11]).

As a particular example consider the "logistic" equation

$$
\left\{\begin{array}{ccc}
-\Delta u=\lambda(x) u-a(x) u^{p} & \text { in } \quad \Omega \\
u=+\infty & \text { on } \partial \Omega,
\end{array}\right.
$$

where $\Omega$ is a $C^{2}$ bounded domain, $p>1$ and $\lambda, a$ are Hölder continuous functions with $a \geq 0$. This problem arises in population dynamics, where the equation

$$
\left\{\begin{array}{ccc}
-\Delta u=\lambda u-a(x) u^{p} & \text { in } \quad \Omega_{0} \\
u=0 & \text { on } \partial \Omega_{0}
\end{array}\right.
$$

is considered, being now $\lambda \in \mathbb{R}, \Omega_{0}$ a $C^{2}$ bounded domain and $a$ is assumed to verify $a>0$ in $\Omega \subset \bar{\Omega} \subset \Omega_{0}, a=0$ in $\Omega_{0} \backslash \bar{\Omega}$. In this case, it is shown in [9], [10] and [17] that problem (1.1) determines the asymptotic profile of the solutions to (1.2) in $\Omega$.

This kind of phenomenology has been studied recently for Lotka-Volterra systems of competitive type in [7], [8], [18] and predator-prey type in [3]. However little effort has been directed to the study of large solutions to systems (see [12] for an example with infinitely many solutions).

Our aim in this paper is to analyze positive large solutions to cooperative Lotka-Volterra systems of the form

$$
\left\{\begin{array}{ccc}
-\Delta u=\lambda u-u^{2}+b u v & \text { in } & \Omega \\
-\Delta v=\mu v-v^{2}+c u v & \text { in } & \Omega \\
u=v=+\infty & \text { on } & \partial \Omega
\end{array}\right.
$$

where $\Omega$ is a $C^{2}$ bounded domain, $\lambda, \mu \in \mathbb{R}$ and $b, c>0$. By a solution to (1.3) we mean a pair $(u, v) \in C^{2}(\Omega)^{2}$ such that $u(x), v(x) \rightarrow+\infty$ as $d(x) \rightarrow 0$. This system has been treated for instance in [4], [16] and [19] in the case of homogeneous Dirichlet boundary conditions. It is clear from the results in these works that the size of $b c$ is determinant in the issues of existence of solutions. 
Our first result reveals that this is indeed the situation when dealing with problem (1.3), and also provides with exact asymptotic estimates for the solutions near the boundary. Notice that the relative position of $\lambda$ and $\mu$ with respect to certain principal eigenvalues involving the semitrivial solutions is irrelevant when the boundary conditions are not homogeneous, unlike the case with homogeneous boundary conditions where it is known to play an essential rôle in existence, [4], [16], [19].

Theorem 1.1 Let $\lambda, \mu \in \mathbb{R}$ and $b, c>0$. Then if $b c \geq 1$, problem (1.3) has no positive solutions, while if $b c<1$, there exists at least a positive solution. Moreover, all positive solutions $(u, v)$ verify

$$
\lim _{x \rightarrow x_{0}} d(x)^{2} u(x)=6 \frac{b+1}{1-b c}, \quad \lim _{x \rightarrow x_{0}} d(x)^{2} v(x)=6 \frac{c+1}{1-b c},
$$

uniformly for $x_{0} \in \partial \Omega$.

It is of course of interest to determine the number of positive solutions to (1.3). It turns out that this is a fairly complicated matter even with finite boundary conditions. So we restrict ourselves to give some necessary conditions which imply uniqueness, as in [4] and [16].

Theorem 1.2 Assume that

$$
\sup _{\Omega}\left(\frac{u}{v}\right) \sup _{\Omega}\left(\frac{v}{u}\right) \leq \frac{1}{b c}
$$

for every positive solution $(u, v)$ to (1.3). Then (1.3) has a unique positive solution.

Condition (1.4) can be somewhat clarified in terms of the data of the problem. If we denote by $\theta_{\lambda}$ the unique positive solution to (1.1) with $a(x) \equiv 1$ and $p=2$ (see Lemma 2.1 in $\S 2$ ), we obtain the following:

Corollary 1.1 Assume $\lambda \geq \mu$ and

$$
\sup _{\Omega}\left(\frac{\theta_{\lambda}}{\theta_{\mu}}\right)^{2} \leq \frac{1}{b c} .
$$

Then problem (1.3) has a unique positive solution. In particular, if $\lambda=\mu$ and $b c<1$, then problem (1.3) always has a unique positive solution. 
It is worthy of mention that the quotient $\theta_{\lambda} / \theta_{\mu}$ is bounded in $\Omega$, thanks to Lemma 2.1 in Section 2, and thus the supremum in (1.5) is always finite.

Alongside with problem (1.3), we briefly treat the case in which one of the boundary conditions is finite, say

$$
\left\{\begin{array}{ccc}
-\Delta u=\lambda u-u^{2}+b u v & \text { in } & \Omega \\
-\Delta v=\mu v-v^{2}+c u v & \text { in } & \Omega \\
u=+\infty, v=\gamma & \text { on } & \partial \Omega
\end{array}\right.
$$

where now $\gamma \geq 0$. By a solution we mean a pair $(u, v) \in C^{2}(\Omega)^{2}$, with $v \in C(\bar{\Omega})$ and $u(x) \rightarrow+\infty$ as $d(x) \rightarrow 0, v(x)=\gamma$ on $\partial \Omega$. With regard to this problem we prove the following nonexistence result.

Theorem 1.3 Let $\lambda, \mu \in \mathbb{R}$ and $b, c>0$. Then if $\gamma>0$, problem (1.6) has no positive solutions. If $\gamma=0$, then there exists $c_{0} \geq 0$ (independent of $\lambda, \mu$ and $b$ ), such that problem (1.6) has no positive solutions if $c>c_{0}$.

Remark 1.1 a) Notice that solutions to (1.3) are always supersolutions to (1.6), while $(0,0)$ is a subsolution. Theorem 1.3 then reveals that the method of sub and supersolutions is not applicable in this setting (compare with Lemma 4 in [11]).

b) The existence of solutions to (1.6) with $\gamma=0$ and $c$ small leads to the study of a logistic problem involving a weight that blows up on the boundary with a critical growth (see Section 9 in [18] and Section 3.2 in [7] for a similar situation). The study of this kind of problems is far from obvious as the existing literature proves (cf. [14]), and will be treated elsewhere.

An outline of the paper is as follows: in Section 2 we recall some known results which will be needed later, which cover the logistic equation (1.1) and the cooperative system with finite boundary conditions. Section 3 is devoted to the existence and nonexistence of solutions to (1.3), while in $\S 4$ we analyze the boundary behaviour and uniqueness of positive solutions. Finally, Section 5 deals with nonexistence of solutions to problem (1.6). Our main tool in the paper is the method of sub and supersolutions, both for single equations and cooperative systems (cf. [22]).

\section{Preliminaries}

In this section we collect some preliminary results which are needed to study the positive solutions to (1.3). The first result concerns the logistic equation with 
blow up on the boundary:

$$
\left\{\begin{array}{ccc}
-\Delta u=\lambda(x) u-u^{2} & \text { in } & \Omega \\
u=+\infty & \text { on } & \partial \Omega .
\end{array}\right.
$$

The following Lemma is a consequence of the results in a number of works, for instance [1], [5], [6], [11], [20], to mention but a few (see the reference list in [11]).

Lemma 2.1 Let $\lambda \in C^{\alpha}(\bar{\Omega})$. Then problem (2.7) has a unique positive solution $\theta_{\lambda}$. Moreover, $\theta_{\lambda}$ is the limit in $C_{\mathrm{loc}}^{2}(\Omega)$ of the increasing family $\left\{\theta_{\lambda, n}\right\}$, unique solutions to

$$
\left\{\begin{array}{ccc}
-\Delta u=\lambda(x) u-u^{2} & \text { in } & \Omega \\
u=n & \text { on } & \partial \Omega .
\end{array}\right.
$$

In addition,

$$
\lim _{x \rightarrow x_{0}} d(x)^{2} \theta_{\lambda}(x)=6
$$

uniformly for $x_{0} \in \partial \Omega$.

The following fundamental result is an adaptation of Lemma 3.2 in [19] to the case of nonhomogeneous boundary conditions. We include the proof for the reader's convenience.

Lemma 2.2 Let $(u, v) \in C^{2}(\bar{\Omega})^{2}$ be a positive solution to the system

$$
\left\{\begin{array}{ccc}
-\Delta u=\sigma u-u^{2}+b u v & \text { in } & \Omega \\
-\Delta v=\sigma v-v^{2}+c u v & \text { in } & \Omega \\
u=K \gamma, v=\gamma & \text { on } & \partial \Omega,
\end{array}\right.
$$

where $\sigma \in \mathbb{R}, \gamma \geq 0$ and $K=(b+1) /(c+1)$. Then $u=K v$. In particular, $v$ is a positive solution to the equation

$$
\left\{\begin{array}{ccc}
-\Delta v=\sigma v-K^{\prime} v^{2} & \text { in } & \Omega \\
v=\gamma & \text { on } & \partial \Omega
\end{array}\right.
$$

with $K^{\prime}=(1-b c) /(c+1)$. Moreover, if $b c<1$, then (2.8) has a unique positive solution, given by

$$
\left(\frac{b+1}{1-b c} \theta_{\sigma, \gamma K^{\prime}}, \frac{c+1}{1-b c} \theta_{\sigma, \gamma K^{\prime}}\right) .
$$

Proof:Let $w=(b+1) v-(c+1) u \in C^{2}(\bar{\Omega}), w=0$ on $\partial \Omega$. A straightforward calculation shows that $-\Delta w+(u+v-\sigma) w=0$. On the other hand, we deduce from the first equation in (2.8) that $\lambda_{1}(-\Delta-\sigma+u-b v)>0$, where $\lambda_{1}(-\Delta+m(x))$ stands for the first eigenvalue of $-\Delta+m(x)$ in $\Omega$. Thus we deduce from the monotonicity of this eigenvalue with respect to the weight that $\lambda_{1}(-\Delta-\sigma+u+$ $v)>0$. Hence the maximum principle implies $w \equiv 0$. The remaining assertions in the Lemma are immediate. 


\section{Existence and nonexistence}

We are undertaking in this section the questions of existence and nonexistence of solutions to problem (1.3). The following Lemma contains the first part of Theorem 1.1.

Lemma 3.1 Let $\lambda, \mu \in \mathbb{R}, b, c>0$. If $b c \geq 1$, then system (1.3) has no positive solution, while if $b c<1$, then there exists at least a positive solution.

Proof:Assume $b c \geq 1$, and let $(u, v)$ be a positive solution to (1.3). For $\delta>0$ fixed, let $\Omega_{\delta}=\{x \in \Omega$ : $\operatorname{dist}(x, \partial \Omega)>\delta\}$, and take $\gamma>0$ so that

$$
u \geq K \gamma, \quad v \geq \gamma \quad \text { on } \partial \Omega_{\delta}
$$

With this choice, and setting $\sigma=\min \{\lambda, \mu, 0\}$, it follows that $(u, v)$ is a supersolution to

$$
\left\{\begin{array}{cc}
-\Delta u=\sigma u-u^{2}+b u v & \text { in } \quad \Omega_{\delta} \\
-\Delta v=\sigma v-v^{2}+c u v & \text { in } \quad \Omega_{\delta} \\
u=K \gamma, v=\gamma & \text { on } \partial \Omega_{\delta},
\end{array}\right.
$$

while $(0,0)$ is a subsolution. Thus $(3.9)$ has a solution $\left(u_{\gamma}, v_{\gamma}\right)$ such that $0 \leq$ $u_{\gamma} \leq u, 0 \leq v_{\gamma} \leq v$ in $\Omega_{\delta}$. On the other hand, by Lemma 2.2, we have $u_{\gamma}=K v_{\gamma}$, where $K$ is given in that Lemma, and $v_{\gamma}$ is a positive solution to

$$
\left\{\begin{array}{ccc}
-\Delta v=\sigma v-K^{\prime} v^{2} & \text { in } & \Omega_{\delta} \\
v=\gamma & \text { on } & \partial \Omega_{\delta}
\end{array}\right.
$$

with $K^{\prime} \leq 0$ also defined in Lemma 2.2. Then, $v_{\gamma}$ is a supersolution to the linear problem

$$
\left\{\begin{array}{ccc}
-\Delta v=\sigma v & \text { in } & \Omega_{\delta} \\
v=\gamma & \text { on } & \partial \Omega_{\delta}
\end{array}\right.
$$

while the zero function is a subsolution. Notice that this problem has a unique solution since $\sigma \leq 0$. Thus $v_{\gamma} \geq \gamma \phi_{\delta}$, where $\phi_{\delta}$ is the unique solution to

$$
\left\{\begin{array}{ccc}
-\Delta v=\sigma v & \text { in } & \Omega_{\delta} \\
v=1 & \text { on } & \partial \Omega_{\delta}
\end{array}\right.
$$

(observe that $0<\phi_{\delta} \leq 1$ in $\Omega_{\delta}$ ). Now letting $\delta \rightarrow 0$, we have that $\phi_{\delta} \rightarrow \phi$, where $\phi$ is the solution to (3.10) in $\Omega$, and we arrive at $u \geq K \gamma \phi, v \geq \gamma \phi$ in $\Omega$. Letting $\gamma \rightarrow+\infty$ we reach a contradiction, which shows that no solution exists in this case. 
Now consider the opposite case $b c<1$. For $n \in \mathbb{N}$ consider the problem:

$$
\left\{\begin{array}{ccc}
-\Delta u=\lambda u-u^{2}+b u v & \text { in } & \Omega \\
-\Delta v=\mu v-v^{2}+c u v & \text { in } & \Omega \\
u=K n, v=n & \text { on } & \partial \Omega .
\end{array}\right.
$$

For $n=n_{0}$ fixed, this problem has at least a solution $\left(u_{n}, v_{n}\right)$, since $(0,0)$ is a subsolution and $(M, N)$, is a supersolution, where $M, N>0$ are large constants such that $M \geq \max \left\{\lambda+b N, K n_{0}\right\}, N \geq \max \left\{\mu+c M, n_{0}\right\}$. Now consider $n=n_{0}+1$. We take $\left(u_{n}, v_{n}\right)$ as subsolution and $(M, N)$ as supersolution and obtain a solution to (3.11) with $n=n_{0}+1$. Proceeding in this way, we construct an increasing sequence of solutions $\left(u_{n}, v_{n}\right)$.

Let us see that this sequence is locally bounded. Indeed, if we let $\sigma=$ $\max \{\lambda, \mu\}$, it follows that $\left(u_{n}, v_{n}\right)$ is a subsolution to

$$
\left\{\begin{array}{ccc}
-\Delta u=\sigma u-u^{2}+b u v & \text { in } & \Omega \\
-\Delta v=\sigma v-v^{2}+c u v & \text { in } & \Omega \\
u=K n, v=n & \text { on } & \partial \Omega,
\end{array}\right.
$$

while large constants $(M, N)$ are again a supersolution. On the other hand, Lemma 2.2 implies that (3.12) has a unique solution

$$
\left(\frac{b+1}{1-b c} \theta_{\sigma, K^{\prime} n}, \frac{c+1}{1-b c} \theta_{\sigma, K^{\prime} n}\right) .
$$

It follows from the preceding discussion and Lemma 2.1 that

$$
\begin{aligned}
& u_{n} \leq \frac{b+1}{1-b c} \theta_{\sigma, K^{\prime} n} \leq \frac{b+1}{1-b c} \theta_{\sigma} \\
& v_{n} \leq \frac{c+1}{1-b c} \theta_{\sigma, K^{\prime} n} \leq \frac{c+1}{1-b c} \theta_{\sigma} .
\end{aligned}
$$

Thus a standard bootstrapping argument gives that $u_{n} \rightarrow u, v_{n} \rightarrow v$ in $C_{\mathrm{loc}}^{2}(\Omega)$, where $(u, v)$ is a solution to (1.3). This completes the proof of the Lemma.

\section{Boundary behaviour and uniqueness}

We are now performing an analysis of the behaviour of the solutions near $\partial \Omega$ in terms of the distance function $d(x)=\operatorname{dist}(x, \partial \Omega)$. We remark that if $\Omega$ is $C^{2}$ then there exists $\delta_{0}>0$ such that $d(x)$ is $C^{2}$ in $0 \leq d(x) \leq \delta_{0}$, and $|\nabla d(x)|=1$ (cf. Lemma 14.16 in [13]). 
We begin by constructing suitable sub and supersolutions to (1.3) near $\partial \Omega$, in the spirit of [5] and [6]. We remark that the construction of the subsolutions is much more delicate than that of the supersolutions.

Lemma 4.1 Assume $b c<1$. Let $A=6(b+1) /(1-b c), B=6(c+1) /(1-b c)$, $c<\eta<1 / b$. Then for every small $\varepsilon>0$, there exist $0<\bar{\delta} \leq \delta_{0}$ and $t_{0}>0$ such that for $0<\delta<\bar{\delta}$ and $t \geq t_{0}$ the functions

$$
\bar{u}(x)=(A+\varepsilon)(d(x)-\delta)^{-2}+K t, \quad \bar{v}(x)=(B+\eta \varepsilon)(d(x)-\delta)^{-2}+t
$$

are supersolutions to (1.3) in $\delta<d(x)<\bar{\delta}$. In the same way, for every small $\varepsilon$, there exist $\bar{\delta}$ and $t_{0}>0$ such that for $0<\delta<\bar{\delta}$, the functions

$$
\underline{u}(x)=(A-\varepsilon)(d(x)+\delta)^{-2}-K t_{0}, \quad \underline{v}(x)=(B-\eta \varepsilon)(d(x)+\delta)^{-2}-t_{0}
$$

are subsolutions to (1.3) in $0<d(x)<\bar{\delta}$ and $\underline{u}(x), \underline{v}(x) \leq 0$ in $\{x \in \Omega: d(x)=$ $\bar{\delta}\}$.

Proof:A somewhat lengthy calculation shows that whenever $\delta<d(x)<\delta_{0}$,

$$
\begin{aligned}
(d & -\delta)^{4}\left(-\Delta \bar{u}-\lambda \bar{u}+\bar{u}^{2}-b \bar{u} \bar{v}\right) \\
& =\varepsilon(A+\varepsilon)(1-b \eta)+2(A+\varepsilon)(d-\delta) \Delta d \\
& +(d-\delta)^{2}\left\{\left[\left(12 K+6 B^{-1} \varepsilon+K(1-b \eta) \varepsilon\right] t-\lambda(A+\varepsilon)\right\}\right. \\
& +(d-\delta)^{4} K t\left(6 B^{-1} t-\lambda\right)
\end{aligned}
$$

Thus for $\varepsilon>0$ fixed we can find $t_{0}>0$ such that the last two expressions are positive if $t \geq t_{0}$. Hence

$$
\begin{aligned}
& (d-\delta)^{4}\left(-\Delta \bar{u}-\lambda \bar{u}+\bar{u}^{2}-b \bar{u} \bar{v}\right) \\
& \quad \geq(A+\varepsilon)\left[(1-b \eta) \varepsilon-2 \bar{\delta} \sup _{d \leq \delta_{0}}|\Delta d|\right]>0,
\end{aligned}
$$

provided $\bar{\delta}=\bar{\delta}(\varepsilon)$ is chosen small enough. A similar calculation shows that $-\Delta \bar{v}-\mu \bar{v}+\bar{v}^{2}-c \bar{u} \bar{v}>0$ if $\delta<d(x)<\bar{\delta}$.

As for the subsolution, proceeding as before, we have that

$$
\begin{aligned}
& (d+\delta)^{4}\left(-\Delta \underline{u}-\lambda \underline{u}+\underline{u}^{2}-b \underline{u v}\right) \\
& \quad=-\varepsilon(A-\varepsilon)(1-b \eta)+2(A-\varepsilon)(d+\delta) \Delta d-\lambda(A-\varepsilon)(d+\delta)^{2} \\
& \quad+(d+\delta)^{4} t\left(6 K B^{-1} t+\lambda K+(d+\delta)^{-2}\left(-12 K+\varepsilon 6 B^{-1}+\varepsilon K(1-b \eta)\right)\right) .
\end{aligned}
$$


The last expression between brackets is negative in $0<d(x)<\bar{\delta}$ provided that

$$
t \leq \frac{(\delta+\bar{\delta})^{-2}\left(12 K-\varepsilon 6 B^{-1}-\varepsilon K(1-b \eta)\right)-\lambda K}{6 K B^{-1}} .
$$

Thus, choosing a value of $t$ verifying this inequality and then taking $\bar{\delta}$ small enough, it follows that $-\Delta \underline{u}-\lambda \underline{u}+\underline{u}^{2}-b \underline{u v}<0$ if $0<d(x)<\delta<\bar{\delta}$.

On the other hand, if we further restrict $t$ to verify

$$
t \geq \frac{(A-\varepsilon)(\delta+\bar{\delta})^{-2}}{K}
$$

we achieve $\bar{u} \leq 0$ if $d(x)=\bar{\delta}$. It is then sufficient to have

$$
\frac{(A-\varepsilon)(\delta+\bar{\delta})^{-2}}{K} \leq \frac{(\delta+\bar{\delta})^{-2}\left(12 K-\varepsilon 6 B^{-1}-\varepsilon K(1-b \eta)\right)-\lambda K}{6 K B^{-1}}
$$

which is certainly possible if $\varepsilon$ and $\bar{\delta}$ are small.

A similar calculation for $\underline{v}$ shows that $t$ must be taken to verify as well:

$$
(B-\eta \varepsilon)(\delta+\bar{\delta})^{-2} \leq t \leq \frac{(\delta+\bar{\delta})^{-2}(12+O(\varepsilon))-\mu}{6 B^{-1}}
$$

which is also possible. A convenient value for $t$ is $t=3 B(\delta+\bar{\delta})^{-2} / 2$. This finishes the proof of the Lemma.

Next we use the sub and supersolutions given by Lemma 4.1 to ascertain the exact behaviour of the solutions near the boundary. Notice that this result, together with Lemma 3.1 completes the proof of Theorem 1.1.

Lemma 4.2 Assume bc $<1$. If $(u, v)$ is a positive solution to (1.3), then

$$
\lim _{x \rightarrow x_{0}} d(x)^{2} u(x)=6 \frac{b+1}{1-b c}, \quad \lim _{x \rightarrow x_{0}} d(x)^{2} v(x)=6 \frac{c+1}{1-b c},
$$

uniformly for $x_{0} \in \partial \Omega$. In particular, if $\left(u_{1}, v_{1}\right)$ and $\left(u_{2}, v_{2}\right)$ are two positive solutions to (1.3), then

$$
\lim _{d(x) \rightarrow 0} \frac{u_{1}(x)}{u_{2}(x)}=\lim _{d(x) \rightarrow 0} \frac{v_{1}(x)}{v_{2}(x)}=1 .
$$

Proof:Let $(u, v)$ be a positive solution to (1.3), and fix $\varepsilon>0$ small. Define $\Omega^{\delta}=$ $\{x \in \Omega: \delta<d(x)<\bar{\delta}\}$, where $\bar{\delta}$ is given in Lemma 4.1, and set $\sigma=\max \{\lambda, \mu\}$. We now choose an arbitrary function $v_{0}$ such that

$$
u \leq K v_{0}, \quad v \leq v_{0} \quad \text { on } \partial \Omega^{\delta}
$$


Notice that $\partial \Omega^{\delta}$ has a component not depending on $\delta$, given by $d(x)=\bar{\delta}$. Thus we can fix $v_{0}$ on $d(x)=\bar{\delta}$. It turns out that $(u, v)$ is a subsolution to the problem

$$
\left\{\begin{array}{ccc}
-\Delta u=\sigma u-u^{2}+b u v & \text { in } \quad \Omega^{\delta} \\
-\Delta v=\sigma v-v^{2}+c u v & \text { in } \quad \Omega^{\delta} \\
u=K v_{0}, v=v_{0} & \text { on } \partial \Omega^{\delta},
\end{array}\right.
$$

and we get as before $u \leq u_{\delta}, v \leq v_{\delta}$, where $\left(u_{\delta}, v_{\delta}\right)$ is the unique positive solution to $(4.14)$.

On the other hand, we can choose $t$ large enough so that $\bar{u} \geq K v_{0}, \bar{v} \geq v_{0}$ on $d(x)=\bar{\delta}$, where $(\bar{u}, \bar{v})$ is the supersolution given by Lemma 4.1. It follows that $\bar{u} \geq u_{\delta}, \bar{v} \geq v_{\delta}$ in $\Omega^{\delta}$ (notice that $\bar{u}=\bar{v}=+\infty$ in $d(x)=\delta$, but this problem can be easily overcome by considering (4.14) in $\Omega^{\tau}$ for $\tau>\delta$ and then letting $\tau \rightarrow \delta$ ). To summarize, we obtain

$$
u(x) \leq(A+\varepsilon)(d(x)-\delta)^{-2}+K t, \quad v(x) \leq(B+\eta \varepsilon)(d(x)-\delta)^{-2}+t
$$

if $\delta<d(x)<\bar{\delta}$. Since $t$ is independent of $\delta$, we can let $\delta \rightarrow 0, d(x) \rightarrow 0$ and $\varepsilon \rightarrow 0$ to arrive at

$$
\limsup _{d(x) \rightarrow 0} d(x)^{2} u(x) \leq A, \quad \limsup _{d(x) \rightarrow 0} d(x)^{2} v(x) \leq B
$$

To get the lower estimate we proceed in an analogue fashion, taking as subsolution $\left(\underline{u}_{0}, \underline{v}_{0}\right)$, given by $\underline{u}_{0}=\max \{0, \underline{u}\}, \underline{v}_{0}=\max \{0, \underline{v}\}$, with the value of $t_{0}$ provided by Lemma 4.1 .

It is clear from the above arguments that the limits hold uniformly. Estimate (4.13) is an easy consequence of the preceding discussion. This proves the Lemma.

We now proceed to the proof of Theorem 1.2, following the ideas in [16] (see also [4]).

Proof of Theorem 1.2: Let $\left(u_{1}, v_{1}\right),\left(u_{2}, v_{2}\right)$ be two positive solutions to (1.3). Then, in virtue of (4.13), for $\varepsilon>0$ fixed there exists $\delta_{0}$ such that for every $\delta<\delta_{0}$,

$$
u_{1} \leq(1+\varepsilon) u_{2}, \quad v_{1} \leq(1+\eta \varepsilon) v_{2} \quad \text { in } \Omega \backslash \Omega_{\delta},
$$

where $\Omega_{\delta}=\{x \in \Omega: d(x)>\delta\}$, and $\eta>0$ is fixed, to be chosen below.

We claim that, with a convenient choice of $\eta$, and for $t \geq \varepsilon$, the functions

$$
\left(u_{t}, v_{t}\right)=\left((1+t) u_{2},(1+\eta t) v_{2}\right)
$$


are supersolutions to the problem

$$
\left\{\begin{array}{ccc}
-\Delta u=\lambda u-u^{2}+b u v & \text { in } & \Omega_{\delta} \\
-\Delta v=\mu v-v^{2}+c u v & \text { in } & \Omega_{\delta} \\
u=u_{1}, v=v_{1} & \text { on } & \partial \Omega_{\delta} .
\end{array}\right.
$$

Indeed a straightforward calculation shows that this is the case provided that $u_{2} \geq b \eta v_{2}$ and $\eta v_{2} \geq c u_{2}$. Thus it suffices to take $\eta$ so that

$$
c \frac{u_{2}}{v_{2}} \leq \eta \leq \frac{1}{b} \frac{u_{2}}{v_{2}}
$$

which is always possible on account of (1.4). Since $u_{t} \geq u_{1}, v_{t} \geq v_{1}$ for $t$ large, it follows from the sweeping principle in [16] that

$$
(1+\varepsilon) u_{2} \geq u_{1}, \quad(1+\eta \varepsilon) v_{2} \geq v_{1} \quad \text { in } \Omega_{\delta} .
$$

Hence (4.15) implies that this inequality is valid throughout $\Omega$. Letting $\varepsilon$ go to zero we arrive at $u_{2} \geq u_{1}, v_{2} \geq v_{1}$, and the symmetric argument proves that $u_{2}=u_{1}, v_{2}=v_{1}$, giving the uniqueness.

Proof of Corollary 1.1: Let us prove that condition (1.5) implies (1.4). For this sake, we are estimating all possible solutions $(u, v)$ to $(1.3)$ from above and from below.

Assume $\lambda \geq \mu$, and let $(u, v)$ be a positive solution to (1.3). Notice that, as a consequence of Lemma 4.2, we have

$$
\lim _{d(x) \rightarrow 0} \frac{u(x)}{v(x)}=K .
$$

Define $w=u /(K v)$, and notice that extending $w$ by 1 on $\partial \Omega$, we have $w \in$ $C^{2}(\Omega) \cap C(\bar{\Omega})$. It is not hard to see that $-v \Delta w-2 \nabla v \nabla w=(\lambda-\mu) v w+(b+$ 1) $v^{2} w(1-w)$ in $\Omega$. Assume $\widetilde{\Omega}=\{x \in \Omega: w(x)<1\}$ is nonempty. Then in $\widetilde{\Omega}$ it holds $-v \Delta w-2 \nabla v \nabla w \geq 0$, and the maximum principle implies $w \geq \inf _{\partial \widetilde{\Omega}} w=1$ in $\widetilde{\Omega}$ (notice that the coefficients of the operator are locally bounded, which is all we need for this conclusion). This contradiction proves $w \geq 1$, that is $u \geq K v$. Hence from (1.3), we obtain

$$
-\Delta u \leq \lambda u-\frac{1-b c}{b+1} u^{2} \quad \text { in } \quad \Omega
$$

Thus $(1-b c) /(b+1) u$ is a subsolution to problem (2.7). Also, $M \theta_{\lambda}$ is a supersolution for $M>1$, and $M \theta_{\lambda} \geq(1-b c) /(b+1) u$ in $\Omega$ for $M$ large (observe that 
$\theta_{\lambda} / u$ is bounded in $\Omega$ thanks to Lemmas 2.1 and 4.2). Thus Lemma 4 in [11], the uniqueness of solutions to (2.7) and a similar argument for $v$ lead to

$$
u \leq \frac{b+1}{1-b c} \theta_{\lambda}, \quad v \geq \frac{c+1}{1-b c} \theta_{\mu} \quad \text { in } \Omega .
$$

Since $u \geq K v$, we also have

$$
u \geq \frac{b+1}{1-b c} \theta_{\mu}, \quad v \leq \frac{c+1}{1-b c} \theta_{\lambda} \quad \text { in } \Omega .
$$

We finally combine this two inequalities to get

$$
\sup _{\Omega}\left(\frac{u}{v}\right) \sup _{\Omega}\left(\frac{v}{u}\right) \leq \sup _{\Omega}\left(\frac{\theta_{\lambda}}{\theta_{\mu}}\right)^{2},
$$

and the proof of the corollary is finished with the aid of Theorem 1.2.

Remark 4.1 An alternative proof of the uniqueness can be given when $\lambda=\mu$ : indeed a similar argument as the one used in the proof of Corollary 1.1 gives $u \leq K v$, hence $u=K v$. It follows from Lemma 2.2 that $u=(b+1) /(1-b c) \theta_{\lambda}$, $v=(c+1) /(1-b c) \theta_{\mu}$, which gives the uniqueness.

\section{Proof of Theorem 1.3}

This section is devoted to the proof of Theorem 1.3. That is, we are showing nonexistence of positive solutions to

$$
\left\{\begin{array}{ccc}
-\Delta u=\lambda u-u^{2}+b u v & \text { in } & \Omega \\
-\Delta v=\mu v-v^{2}+c u v & \text { in } & \Omega \\
u=+\infty, v=\gamma & \text { on } & \partial \Omega
\end{array}\right.
$$

when $\gamma>0$ or $\gamma=0$ and $c$ is large enough. The nonexistence proof for $\gamma>0$ is a consequence of the following general Lemma.

Lemma 5.1 Let $\Omega \subset \mathbb{R}^{N}$ be a bounded domain, $f \in C^{\alpha}(\Omega)$ such that $f(x) \sim$ $C_{0} d(x)^{-\beta}$ as $x \rightarrow x_{0}$, for some $x_{0} \in \partial \Omega$, where $C_{0}>0$ and $\beta \geq 2$. Then for every solution $u \in C^{2}(\Omega)$ to $-\Delta u=f(x)$ which is bounded from below, we have

$$
\lim _{x \rightarrow x_{0}} u(x)=+\infty
$$


Proof:Assume $\gamma=\liminf _{x \rightarrow x_{0}} u(x)$ is finite. Then for every $\varepsilon>0$, there exists a neighbourhood $\mathcal{U}$ of $x_{0}$ (relative to $\Omega$ ) such that $u \geq \gamma-\varepsilon$ in $\mathcal{U}$. By diminishing this neighbourhood if necessary, we can achieve $f(x) \geq\left(C_{0}-\varepsilon\right) d(x)^{-\beta}$ in $\mathcal{U}$. Take a sequence $x_{n} \rightarrow x_{0}$ such that $u\left(x_{n}\right) \rightarrow \gamma$. If we denote by $B_{n}$ the ball of center $x_{n}$ and radius $d\left(x_{n}\right)$, we have $B_{n} \subset \mathcal{U}$ for $n$ large, so that $u$ verifies

$$
\left\{\begin{array}{cc}
-\Delta u \geq\left(C_{0}-\varepsilon\right) 2^{-\beta} d\left(x_{n}\right)^{-\beta} & \text { in } \quad B_{n} \\
u \geq \gamma-\varepsilon & \text { on } \partial B_{n}
\end{array}\right.
$$

since $d(x) \leq 2 d\left(x_{n}\right)$ in $B_{n}$. It follows from the maximum principle and a suitable rescaling that

$$
u(x) \geq \gamma-\varepsilon+\left(C_{0}-\varepsilon\right) 2^{-\beta} d\left(x_{n}\right)^{2-\beta} v\left(d\left(x_{n}\right)^{-1}\left(x-x_{n}\right)\right),
$$

where $v$ is the unique solution to

$$
\left\{\begin{array}{ccc}
-\Delta v=1 & \text { in } & B \\
v=0 & \text { on } & \partial B
\end{array}\right.
$$

and $B$ is the unit ball (notice that $v>0$ in $B$ ). Setting $x=x_{n}$ in (5.17) and making $n \rightarrow+\infty$ we directly arrive at a contradiction if $\beta>2$. When $\beta=2$, we also reach a contradiction by letting $n \rightarrow+\infty$ and then $\varepsilon \rightarrow 0$.

Remark 5.1 a) Lemma 5.1 admits obvious generalizations, for instance to the case $C_{0}<0$ and $u$ bounded from above.

b) In the complementary case $0<\beta<2$, bounded solutions to $-\Delta u=f(x)$ may exist, as shown in $\S 6.5$ of [13].

Proof of Theorem 1.3: Assume $\gamma>0$, and let $(u, v)$ be a positive solution to (1.6). Then, since $v \in C(\bar{\Omega})$, it follows that $u$ is a positive solution to

$$
\left\{\begin{array}{ccc}
-\Delta u=(\lambda+b v) u-u^{2} & \text { in } & \Omega \\
u=+\infty & \text { on } & \partial \Omega .
\end{array}\right.
$$

and from Lemma 2.1 we obtain $u=\theta_{\lambda+b v}, u(x) \sim 6 d(x)^{-2}$ as $d(x) \rightarrow 0$. As a consequence, letting $f:=\mu v-v^{2}+c u v$, we have $f(x) \sim 6 c \gamma d(x)^{-2}$ as $d(x) \rightarrow 0$, and $-\Delta v=f(x)$. Thus Lemma 5.1 implies $\lim _{x \rightarrow x_{0}} v(x)=+\infty$, for every $x_{0} \in \partial \Omega$, which contradicts $v=\gamma$ on $\partial \Omega$.

Now assume $\gamma=0$. Lemma 5.1 can not be applied, since $f(x)$ does not behave like $d(x)^{-2}$. Assume for a contradiction that $(u, v)$ is a positive solution to (1.6). Then $u \sim 6 d(x)^{-2}$ as $d(x) \rightarrow 0$. Proceeding as in the first part of the proof, 
together with the reasoning in Lemma 5.1, it follows that in a neighbourhood $\mathcal{U}$ of every point $x_{0} \in \partial \Omega$ we can achieve

$$
\left\{\begin{array}{cc}
-\Delta v \geq \frac{(6 c-\varepsilon)}{4} d\left(x_{n}\right)^{-2} v-v^{2} & \text { in } \quad B_{n} \\
v \geq 0 & \text { on } \partial B_{n}
\end{array}\right.
$$

where $B_{n}$ is the ball of center $x_{n}$ and radius $d\left(x_{n}\right)$, and $x_{n} \rightarrow x_{0}$ is an arbitrary sequence. If we define

$$
\bar{v}(x)=d\left(x_{n}\right)^{2} v\left(x_{n}+d\left(x_{n}\right) x\right),
$$

we have

$$
\left\{\begin{array}{cc}
-\Delta \bar{v} \geq \frac{(6 c-\varepsilon)}{4} \bar{v}-\bar{v}^{2} & \text { in } \quad B \\
\bar{v} \geq 0 & \text { on } \quad \partial B
\end{array}\right.
$$

$B$ standing again for the unit ball. In particular, if $6 c-\varepsilon>4 \lambda_{1}$, where $\lambda_{1}$ is the first eigenvalue of $-\Delta$ in $B$, then $\bar{v} \geq w$, the unique positive solution to

$$
\left\{\begin{array}{cc}
-\Delta w=\frac{(6 c-\varepsilon)}{4} w-w^{2} & \text { in } \quad B \\
w=0 & \text { on } \partial B .
\end{array}\right.
$$

In conclusion, setting $x=0$ in (5.18), we arrive at $v\left(x_{n}\right) \geq d\left(x_{n}\right)^{-2} w(0)$, and we reach again a contradiction letting $n \rightarrow+\infty$. This finishes the proof.

Remark 5.2 Notice in particular that $c_{0} \leq 2 \lambda_{1} / 3$.

\section{References}

[1] C. Bandle and M. Marcus, 'Large' solutions of semilinear elliptic equations: existence, uniqueness and asymptotic behaviour, J. Anal. Math. 58 (1992), $9-24$.

[2] L. Bieberbach, $\Delta u=e^{u}$ und die automorphen Funktionen, Math. Ann. 77 (1916), 173-212.

[3] N. Dancer and Y. Du, Effects of certain degeneracies in the predator-prey model, SIAM J. Math. Anal. 34 (2002), 292-314.

[4] M. Delgado, J. López-Gómez and A. Suárez, On the symbiotic LotkaVolterra model with diffusion and transport effects, J. Diff. Eqns. 160 (2000), 175-262. 
[5] M. Del Pino and R. Letelier, The influence of domain geometry in boundary blow-up elliptic problems, Nonlinear Anal. 48 (2002), 897-904.

[6] G. Díaz and R. Letelier, Explosive solutions of quasilinear elliptic equations: existence and uniqueness, Nonlinear Anal. 20 (1993), 97-125.

[7] Y. Du, Effects of a degeneracy in the competition model. Part I: classical and generalized steady-state solutions, J. Diff. Eqns. 181 (2002), 92-132.

[8] Y. Du, Effects of a degeneracy in the competition model. Part II: perturbation and dynamical behaviour, J. Diff. Eqns. 181 (2002), 133-164.

[9] Y. Du and Q. Huang, Blow-up solutions for a class of semilinear elliptic and parabolic equations, SIAM J. Math. Anal. 31 (1999), 1-18.

[10] J. García-Melián, R. Gómez-Reñasco, J. López-Gómez and J. Sabina de Lis, Point-wise growth and uniqueness of positive solutions for a class of sublinear elliptic problems where bifurcation from infinity occurs, Arch. Rat. Mech. Anal. 145 (3) (1998), 261-289.

[11] J. García-Melián, R. Letelier-Albornoz and J. Sabina de Lis, Uniqueness and asymptotic behaviour for solutions of semilinear problems with boundary blow-up, Proc. Amer. Math. Soc. 129 (2001), 3593-3602.

[12] J. García-Melián, R. Letelier-Albornoz and J. Sabina de Lis, The solvability of an elliptic system under a singular boundary condition, preprint.

[13] D. Gilbarg and N.S. Trudinger, Elliptic partial differential equations of second order, Springer-Verlag, 1983.

[14] J. Hernández, F. Mancebo and J.M. Vega de Prada, On the linearization of some singular nonlinear elliptic problems and applications, to appear in Ann. Inst. H. Poincaré Anal. Non Linéaire.

[15] J.B. Keller, On solutions of $\Delta u=f(u)$, Comm. Pure Appl. Math. 10 (1957), 503-510.

[16] P. Korman and A. Leung, On the existence and uniqueness of positive steady states in the Volterra-Lotka ecological models with diffusion, Appl. Anal. 26 (1987), 145-160.

[17] J. López-Gómez, Large solutions, metasolutions, and asymptotic behaviour of the regular positive solutions of sublinear parabolic problems, El. J. Diff. Eqs., Conf. 05 (2000), 135-171. 
[18] J. López-Gómez, Existence and metacoexistence states in competing species models, to appear in Houston J. Math.

[19] Y. Lou, Necessary and sufficient condition for the existence of positive solutions of certain cooperative systems, Nonlinear Anal. 25 (1994), 1079-1095.

[20] M. Marcus and L. Véron, Uniqueness and asymptotic behaviour of solutions with boundary blow-up for a class of nonlinear elliptic equations, Ann. Inst. H. Poincaré Anal. Non Linéaire 14 (2) (1997), 237-274.

[21] R. Osserman, On the inequality $\Delta u \geq f(u)$, Pacific J. Math. 7 (1957), 1641-1647.

[22] C.V. Pao, Nonlinear parabolic and elliptic equations, Plenum Press, New York, 1992. 